\title{
High-Performance Computing
}

$\mathrm{H}$ igh-performance computing (IIPC) refers to the ability to process data and perform complex calculations at high speeds. It is one of the most essential tools fueling the advancement of science and technology. By aggregating the computing power, IIPC: has found its applications in biosciences, engineering calculations, climate modeling. geographical data collection, and many other fields.

Scientists from the CIS Institute of Computing Technology (ICT) have scored many accomplishments that promote IIPC: in China. In 1983. ICT scientists. together with their collaborators from over 80 units in China, developed and manufactured the Model 75? large-scale computer rated at 10 megaflops (million floating point operations per second). Nodel 7.57 is the first large-scale vector computer system designed and produced in China. winning the first prize of the National Science and Technology Progress Award in 1985.

In 1995. ICT scientists made many technical breakthroughs in large-scale parallel processing and successfully developed the Dawning-1000 massively parallel processor (MNP) with 36 processing nodes and 2.5 gigaflops peak performance, winning the first prize of the National Science and Techonology Progress Award in 1997. In 200t. they developed a more powerful system Dawning-+000 with 10 teraflops peak performance, entitling China s IPC technology and industry to rank among the global Top 10. This achievement won the Chinese Academy of Sciences Outstanding Scientific and Technological Achievement Iward in 2005. Dawning-50004. a later model of the Dawning series developed in 2008. scored the $10^{\text {th }}$ position on the list of the $32^{\text {th }}$ TOP500 Ciobal I lighPerformance (omputer Ranking.

In 2010. ICT scientists successfully released the Dawning Nebulae Supercomputer (a partition of Dawning 6000). which was the first supercomputer with measured performance over 1 petaflops. Dawning Nebulae Supercomputer was ranked the second fastest supercomputers in the world in the $3.5^{-1 \text { th }}$ TOP.500 list

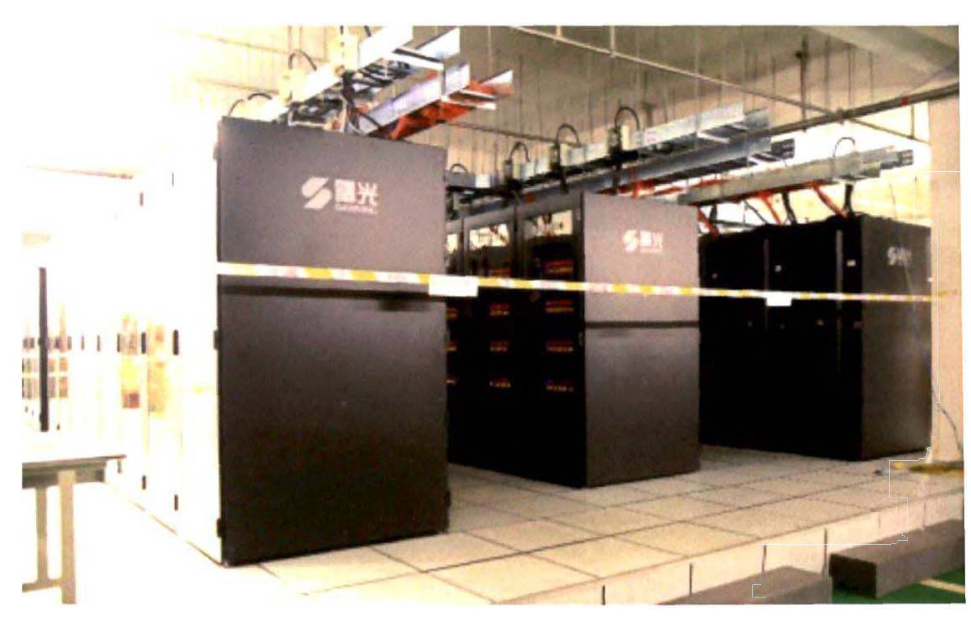

Dawning-5000A. (Credit: ICT)

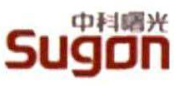

曙光星云高效能计算机系统

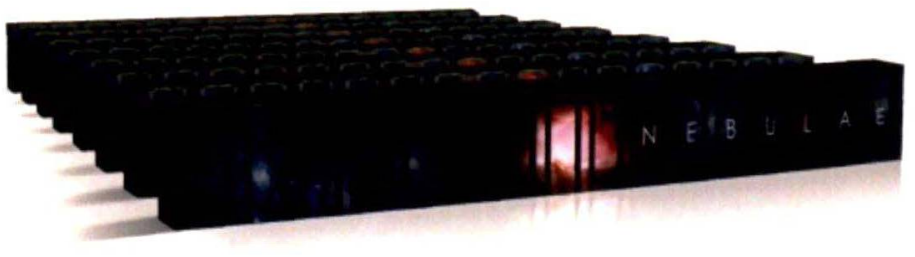

The Dawning Nebulae Supercomputer with measured performance of 1.27 petaflops. (Credit: ICT)

released in June. 2010. which was the highest ranking ever achieved by a supercomputer made in (hina. From 2009 to 2016. Dawning series supercomputers ranked the first in China's high-performance computer market for eight consecutive years.

Scientists at the lnstitute of Software of the Chinese Academy of Sciences (ISCAS) have also made their landmark contributions to IIPC in China. They have conmitted to software development for China shigh- 


\section{DD-MG k-cycle}

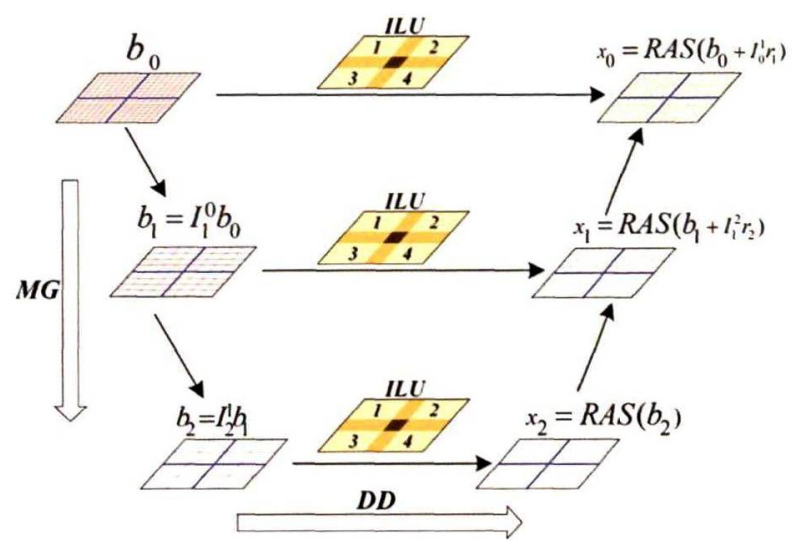

Process-level parallellism: $\mathrm{O}(100 \mathrm{k})$

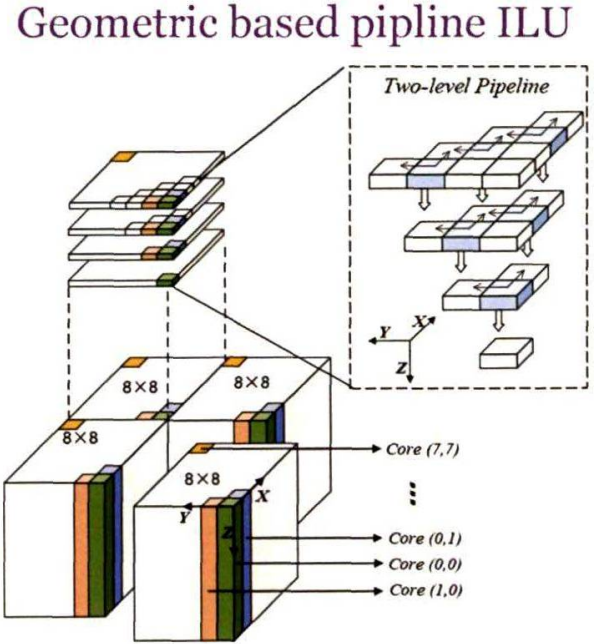

Thread-level parallellism: $\mathrm{O}(100)$

The kernel algorithm for the world's first 10M-core fully-implicit solver. (Credit: ISCAS)

performance computers, such as Dawning, Lenovo. Sunway and Tianhe.

In addition, ISCAS scientists have developed many new algorithms. In particular, they invented a domaindecomposed algorithm that is suitable for dealing with large heterogeneous conditions, such as weatherclimate prediction. The major obstacle to the precise weather-climate prediction lies in dealing with various spatial and temporal scales. Because the atmosphere contains time-dependent multi-scale dynamics. To tackle these challenges, they developed a highly scalable fully implicit solver (method for calculating). which successfully scales to the entire system of the Sunway Taihulight - a Chinese supercomputer with over 10.5 $\mathrm{M}$ heterogeneous cores - and allows for a performance of 7.95 petaflops, winning the 2016 ACM Gordon Bell Prize. Their achievements have also been appreciated by the Academy, winning the Chinese Academy of Sciences Outstanding Scientific and Technological Achievement Award in 2017. 